\title{
Electrically Induced Microstructures in Micro- and Nano-Suspensions and Related Physical Properties
}

\author{
By Katsufumi TANAKA* and Ryuichi AKIYAMA
}

Progress in electrorheology was reviewed for micro- and nano-suspensions. Characteristics required for the suspensions and related materials were shown. Some proposed models were discussed. Electrically induced microstructures, yielding, and related physical properties were also discussed in terms of theory and experiment.

KEY WORDS: Electrorheology / Microstructure / Nanoparticles / Suspensions / Yield Stress /

The electrorheological (ER) effect is the reversible rheological responses of fluids only by application and removal of an external electric field. A suspension composed of microparticles and insulating oil is known to show the ER effect. The micro-suspension is a typical ER fluid, ${ }^{1-14}$ but "homogeneous" ER fluids are also known. ${ }^{15,16}$ The flow of the ER microsuspension under no electric fields is generally assumed to be the Newtonian flow. Under an electric field of $E$, the flow is well assumed to be the Bingham flow with the yield stress depending on $E, \sigma_{\mathrm{y}}(E)$,

$$
\sigma(E)=\eta_{\mathrm{c}} \dot{\gamma}+\sigma_{\mathrm{y}}(E),
$$

where $\sigma(E)$ is the shear stress under the electric field, $\eta_{\mathrm{c}}$ is the viscosity of the continuous phase, and $\dot{\gamma}$ is the shear rate.

In a micro-suspension, randomly distributed primary particles are polarized and a chain-like microstructure along the electric field is induced, ${ }^{2,5-7,10}$ as shown in Figure 1. A columnar or further developed microstructure of micro-particles was also observed. Because a characteristic response time of the ER effect is on the order of milliseconds, ${ }^{11,12}$ there are substantial expectations for applications, ${ }^{1,2,6,11,13,14}$ such as dampers, clutches, valves, robotics, force display devices, and so on.

Electrorheology was extensively reviewed in the 1980s. ${ }^{17,18}$ So far, some conference books ${ }^{19-22}$ as well as review $\operatorname{articles}^{15-18,23,24}$ have also been published. Recently, however, particular attention has been directed toward the ER fluids based on micro- and nano-suspensions, the current understanding of which will be focused on in this review.

\section{CHARACTERISTICS REQUIRED FOR THE ER SUSPENSIONS}

The ER effect for the suspensions is first characterized by the (dynamic or static) yield stress or induced stress, $\sigma(E)-\sigma(0)$ derived from eq 1 . Figure 2 shows a hypothetical stress-strain curve for slow deformations. The dashed line indicates hysteresis, which can occur before the maximum. It is

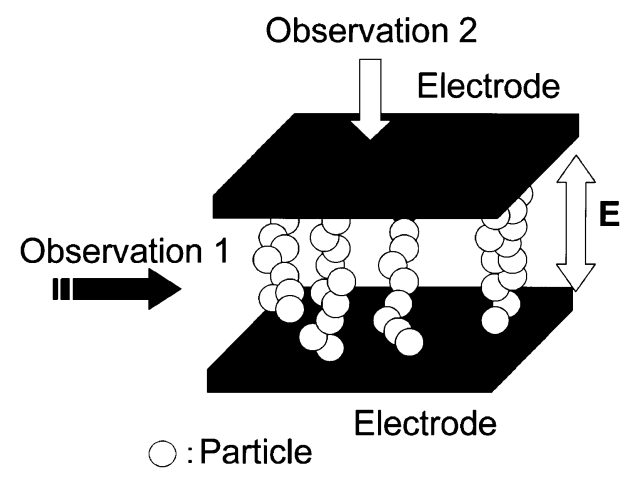

Figure 1. An example of a chain-like microstructure along the electric field in the quiescent state. Typical geometries for optical observations are shown.

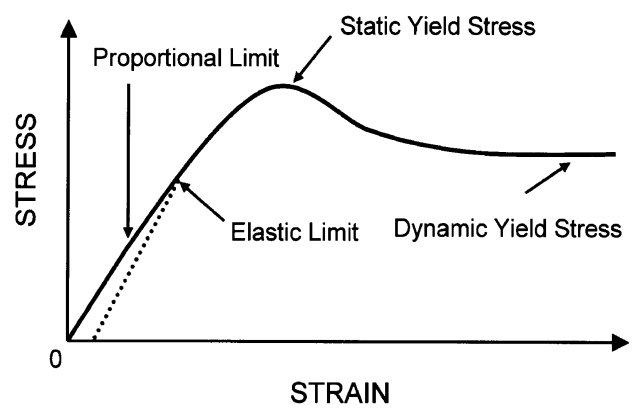

Figure 2. A hypothetical stress-strain curve for slow deformations. The dashed line indicates hysteresis, which can occur before the maximum.

assumed that the static yield stress is not necessarily the same as the dynamic yield stress in the figure..$^{25,26}$ Experimentally, the value or even the existence of a yield stress could depend on the duration of the experiment, and the meaning of the yield stress is not necessarily universal. ${ }^{27,28}$ Further, the stresses for the proportional limit and the elastic limit shown in Figure 2 can be defined as the yield stress.

There are some other characteristics required, ${ }^{17}$ such as stability against the sedimentation of particles, as wide as

Department of Macromolecular Science and Engineering, Graduate School of Science and Technology, Kyoto Institute of Technology, Matsugasaki, Kyoto 606-8585, Japan

*To whom correspondence should be addressed (Tel: +81-(0)75-724-7840, Fax: +81-(0)75-724-7840, E-mail: ktanaka @ kit.ac.jp). 
possible a temperature range of operation, low (or desirable levels of) conductance, and so on. Applicability to a narrow gap is of current interest for micro- or nano-fluidics. Much larger yield stresses are also demanded.

\section{MATERIALS}

Winslow $^{2}$ first reported quantitative data on an ER fluid based on moist silica gel, as well as the observation that wet particulates were most active. Although the composition of ER-active fluids was summarized, ${ }^{17}$ unspecified surfactants or additives were often applied. Assuming an electric double layer of fixed ions and counter ions, ER suspensions based on ionic exchange resin particles were developed by Sugimoto, ${ }^{5}$ and a systematic study was carried out.

For practical applications, however, the presence of water in the fluid is undesirable: it may result in device corrosion; operational temperature limitation to a range somewhere between $-20^{\circ} \mathrm{C}$ and $70{ }^{\circ} \mathrm{C}$; and significant electrical power consumption with thermal instability. ${ }^{17}$ Such a requirement of water has been overcome since dry (or anhydrous) ER suspensions were developed. ${ }^{8,9}$ Anhydrous ER fluids based on poly(acene quinones) were developed by Block et al., ${ }^{8}$ and the fluids based on the family of crystalline alumino-silicates were developed by Filisko et al. $^{9}$

Furthermore, anhydrous ER fluids were developed based on poly( $p$-phenylene $),{ }^{29}$ polyaniline, ${ }^{30}$ and carbonaceous ${ }^{31}$ particles. A new type of anhydrous ER fluids was originally developed by Inoue ${ }^{32}$ based on a conductive core, surfacemodified with a nonconductive thin skin. Anhydrous ER fluids based on composite particles were also reported. ${ }^{33-35}$ For a favorable level of induced stress, a series of surface-modified particles of sulfonated poly(styrene-co-divinyl benzene)s was developed by Asako et al. ${ }^{36}$

Recently, a suspension based on dry titanium dioxide $\left(\mathrm{TiO}_{2}\right)$ nano-particles has been reported by Tanaka et al. ${ }^{37}$ The suspended particles were remarkably stable against sedimentation and electrical breakdown. The nano-suspension also showed a good fluidity with a gap of $50 \mu \mathrm{m}$. An induced stress of over $3 \mathrm{kPa}$ was generated by a DC electric field of $16 \mathrm{kV} \cdot \mathrm{mm}^{-1}$. The ER effect was also investigated for nano-suspensions based on carbon nono-tubes ${ }^{38}$ and fullerenes. ${ }^{39}$ On the other hand, a micro-suspension based on Ce-doped $\mathrm{TiO}_{2}$ particles has been developed by Yin and Zhao. ${ }^{40} \mathrm{An}$ induced stress of $8 \mathrm{kPa}$ by a DC electric field of $4 \mathrm{kV} \cdot \mathrm{mm}^{-1}$ and a favorable level of induced stress at temperatures around $100{ }^{\circ} \mathrm{C}$ were reported. Furthermore, a new class of giant ER effect has been proposed by Wen et ll. $^{41} \mathrm{~A}$ static yield stress as well as an induced stress over $100 \mathrm{kPa}$ was reported for a nano-suspension based on barium titanyl oxalate $\left(\mathrm{BaTiO}\left(\mathrm{C}_{2} \mathrm{O}_{4}\right)_{2}\right)$ coated with urea.

\section{SOME PROPOSED MODELS}

\section{The Forces Relevant to the ER Effect and Their Competi- tion}

A number of physical models including microscopic models

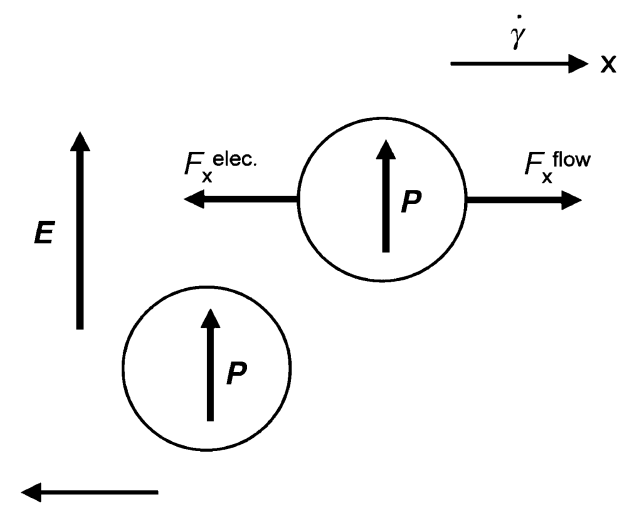

Figure 3. A pair of polarized particles under shear flow. The lateral component of the polarization force $\left(F_{\mathrm{X}}^{\text {elec. }}\right)$ and that of the viscous force $\left(F_{\mathrm{X}}{ }^{\text {flow }}\right)$ acting on the top particle are shown.

of particle-level dynamics have been proposed and reviewed ${ }^{24}$ extensively. Here, some proposed models will be discussed, while microstructures including the ground state of the ER solid $^{42}$ will be discussed later.

The forces relevant to the ER effect and the competition among them were discussed by Marshall et al. ${ }^{43}$ Under an external electric field, polarization forces are induced, which scales as $12 \pi \varepsilon_{\mathrm{c}} \varepsilon_{0}(a \beta E)^{2}$, where $\varepsilon_{0}$ is the dielectric constant of free space, $\beta\left[=\left(\varepsilon_{\mathrm{p}}-\varepsilon_{\mathrm{c}}\right) /\left(\varepsilon_{\mathrm{p}}+2 \varepsilon_{\mathrm{c}}\right)\right]$ is the relative dielectric constant mismatch, $\varepsilon_{\mathrm{c}}$ and $\varepsilon_{\mathrm{p}}$ are respectively the relative dielectric constant of the continuous phase and that of the particles, and $a$ is the particle radius. In Figure 3, a pair of polarized particles under shear flow is shown. (Basically, the polarization is assumed to be instantaneously induced. The models neglecting the conduction current can be categorized as polarization models, ${ }^{24}$ while the models considering this are classified as conduction models. In practice, both the particles and the continuous phase can be weakly conductive.)

Under shear flow, viscous forces also act on the particles with a characteristic scale of $6 \pi \eta_{\mathrm{c}} a^{2} \dot{\gamma}$. In nano-suspensions, thermal forces should be taken into consideration, which scales as $k T / a$ where $k$ is Boltzmann constant, and $T$ is absolute temperature. In real colloidal suspensions, colloidal forces can be important, such as van der Waals attractive forces, which scales as $A / 12 h$, and electrostatic (or ionic) repulsive forces due to the overlap of diffuse double layers, which scales as $4 \pi \varepsilon_{\mathrm{c}} \varepsilon_{0} \Psi_{0}{ }^{2}$, where $A$ is the Hamaker coefficient, $h$ is the minimum particle surface to surface separation, and $\Psi_{0}$ is the surface potential. Steric (or polymeric) repulsive forces may be considered for the surfacemodified particles with a thin polymer layer in suspension. ${ }^{43,44}$ It is worthwhile to note that a model including water capillary forces (or water bridges ${ }^{6}$ ) in water-promoted ER suspensions was proposed by See et al. ${ }^{45}$

Competition among the forces can be evaluated using dimensionless groups ${ }^{43}$ for instance:

[viscous forces]/[polarization forces]

$$
=\eta_{\mathrm{c}} \dot{\gamma} / 2 \pi \varepsilon_{\mathrm{c}} \varepsilon_{0}(\beta E)^{2} \equiv M_{\mathrm{n}}
$$

[thermal forces]/[polarization forces]

$$
=k T / a^{3} 12 \pi \varepsilon_{\mathrm{c}} \varepsilon_{0}(\beta E)^{2}\left(\propto P, \lambda^{-1}\right)
$$


The dimensionless group in eq 2 is often referred to as the Mason number, $M_{\mathrm{n}}$, which is a characteristic ratio of viscous forces and polarization forces acting on a particle. ${ }^{46}$ The dimensionless groups of $P$ and $\lambda^{-1}$ similar to eq 3 are also defined $^{47-50}$ for evaluating the Brownian motion, showing that thermal forces are negligible for most micro-suspensions, while it can be important for nano-suspensions.

\section{Polarization Models}

A droplet model considering aggregated particles in suspension was proposed by Halsey and Toor. ${ }^{49}$ The droplet shape was set by a competition between the field energy and surface tension. The shear thinning was observed in a model with a sheared droplet. ${ }^{50}$

Microstructures under an electric field and related rheological properties were investigated using dynamic simulation methods of particle level. ${ }^{51-55}$ The dynamics of the fibrous nature by Klingenberg et al. ${ }^{51}$ and aggregation kinetics by See and Doi ${ }^{52}$ of non-Brownian suspensions were reported in the quiescent state. The dynamic yield stress was simulated under shear, and configurations of the particles were also shown. ${ }^{53}$ The induced stress due to the clusters (chains or aggregates of the particles) was simulated by Takimoto, ${ }^{54}$ showing that the induced stress remained roughly independent of the shear rate, although the clusters became shorter as the shear rate increased. In a moleculardynamics-like simulation, in which both hydrodynamic and electrostatic interparticle interactions can be accurately accounted for, ${ }^{55}$ the shear thinning was found, and the corresponding microstructures were shown. For small Mason number, the microstructure began to exhibit two distinct motions, a slow elastic body-like deformation and a rapid reconfiguration or snapping of the microstructure (or "energy jumps"), suggesting a close relation to the dynamic yield stress, or energy dissipation.

The static and dynamic yield stresses were described by Bonnecaze and Brady ${ }^{56}$ using a microstructural model, in which both yield stresses are related to the electrostatic energy. The static yield stress was determined from non-linear elastic strain-energy theory. ${ }^{57}$ The connection between the dynamic yield stress and the "energy jumps" 55 was derived. In the polarization models, the dynamic (or static) yield stress is basically proportional to $(\beta E)^{2}$.

\section{Conduction Models}

It was pointed out by Anderson ${ }^{58}$ and Davis ${ }^{59,60}$ that the (Ohmic) conductivities of particles, $\kappa_{\mathrm{p}}$, and continuous phase, $\kappa_{\mathrm{c}}$, are dominant for the polarization forces under DC (or lowfrequency $\mathrm{AC}$ ) electric fields. On the other hand, the dielectric constants of $\varepsilon_{\mathrm{p}}$ and $\varepsilon_{\mathrm{c}}$ are dominant under high-frequency $\mathrm{AC}$ electric fields, ${ }^{60}$ at a frequency $f$ of which the conductivity effects can be neglected (i.e., $\omega=2 \pi f \gg \kappa_{\mathrm{i}} / \varepsilon_{0} \varepsilon_{\mathrm{i}}$, for both particles $(\mathrm{i}=\mathrm{p}$ ) and the continuous phase $(\mathrm{i}=\mathrm{c})$ ). Under DC (or lowfrequency $\mathrm{AC})$ electric fields, $\beta\left[=\left(\kappa_{\mathrm{p}}-\kappa_{\mathrm{c}}\right) /\left(\kappa_{\mathrm{p}}+2 \kappa_{\mathrm{c}}\right)\right]$ can be modified. These effects can be generally treated using complex dielectric constants, ${ }^{60,61} \varepsilon^{*}{ }_{\mathrm{i}}=\varepsilon^{\prime}{ }_{\mathrm{i}}-\kappa_{\mathrm{i}} /\left(j \omega \varepsilon_{0}\right)$, where $j^{2}=-1$, instead of $\varepsilon_{\mathrm{i}}$ or $\kappa_{\mathrm{i}}$ in $\beta$. The frequency dependence of the dynamic yield stress can be qualitatively explained in the models.

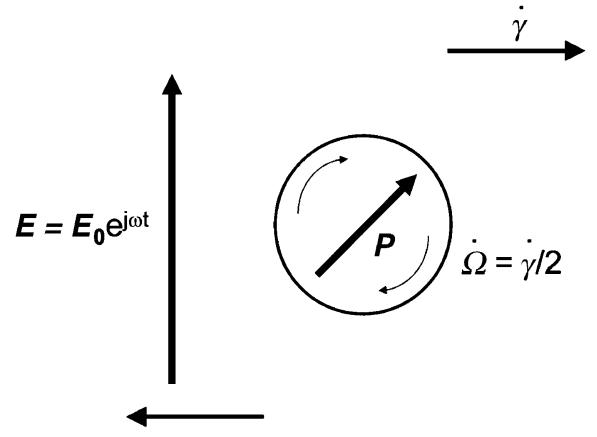

Figure 4. A shear-rate dependent polarization $(\boldsymbol{P})$ of a particle spinning in flow with an $A C$ field $(E)$. A torque $(\boldsymbol{P} \times \boldsymbol{E})$ can also be induced by a delay in the polarization of the spinning particle and a tilt of the polarization $(\boldsymbol{P})$ from the electric field direction $(\boldsymbol{E})$.

For ER suspensions, the conductivity often shows nonOhmic behavior. If the difference between $\kappa_{\mathrm{p}}$ and $\kappa_{\mathrm{c}}$ in $\beta$ is reduced, the electrical force acting between the particles is reduced. The resulting dependence of the induced stress can be weaker than expected. ${ }^{37}$ The non-Ohmic conduction was included by Foulc et $a l .{ }^{62}$ in a model considering the two regions in the contact zone between spherical particles, which can be determined by balancing the conductances through the outer and inner regions. The non-Ohmic behavior was given by a simplified expression of Onsager's theory, ${ }^{63}$ showing to a first approximation the linear attraction force at high electric fields. A different approach was proposed by Tang et al. ${ }^{64}$

\section{The ER Resonance}

It was pointed out by Block et al ${ }^{8,17}$ that the resonance of a shear-rate dependent polarization $(\boldsymbol{P})$ of the particle with an AC field $(\boldsymbol{E})$ can be induced, as shown in Figure 4. A torque $(\boldsymbol{P} \times \boldsymbol{E})$ is then induced by a delay in the polarization of the spinning particle and a tilt of the polarization $(\boldsymbol{P})$ from the electric field $(\boldsymbol{E})$, the torque of which contributes at least in part to the ER effect. In flow-modified permittivity, resonance was observed dielectrically for colloid systems in shear and electric fields. ${ }^{8,17}$ In the ER measurement, resonance has been reported by Negita and Ohsawa. ${ }^{65}$ Simultaneous measurements of the ER effect and the flow-modified permittivity have also been reported by Misono and Negita. ${ }^{66}$ A positive resonance peak was observed in each spectrum of the ER effect and first-order dielectric permittivity, while a negative one was observed in the thirdorder dielectric permittivity, suggesting a shear-induced particle rotation even at high electric fields. The observations of the ER resonance also suggest time-dependent dielectric polarization.

\section{ELECTRICALLY INDUCED MICROSTRUCTURES}

\section{Microstructures in the Quiescent State}

In the droplet model, ${ }^{49,67}$ dielectric particles rapidly form chains or columns spanning the electrodes upon application of a high electric field. Each column has a width $\sim a(L / a)^{2 / 3}$, where $a$ is the radius of a particle and $L$ the distance between electrodes. The chains or columns then slowly drift together by thermal fluctuations with a long-time relaxation (or densifications) for a macroscopic phase separation. 
A three-dimensional microstructure of a body-centered tetragonal (bct) lattice was proposed by Tao and $\mathrm{Sun}^{42}$ as the ground state of the ER solid, which was determined for nonBrownian particles with the dipole limit by analytical argument and energy calculations. The bct lattice was also observed in a simulation $^{68}$ and experiment. ${ }^{69}$ In extensive computer simulations, microstructures were further investigated for Brownian particles. ${ }^{70} \mathrm{~A}$ bct lattice can be developed when the electric field is strong and thermal fluctuations are weak or moderate. In a very strong field with or without very weak thermal fluctuations, a polycrystalline structure consisting of many small bct lattice grains may be developed. A glasslike structure is also developed if both the electric field and thermal fluctuations are strong. In all of the three microstructures, a thick-column structure can be induced.

Furthermore, an electric-field-driven bulk phase transition in a suspension has been reported by Kumar et al. ${ }^{71}$ under a uniform AC electric field where the effects of other competing forces are suppressed. After chain-column formation, a cellular pattern was found consisting of particle-free domains surrounded by particle-rich walls.

\section{Development of Microstructures in the Quiescent State}

In a dynamic simulation by Toor, $^{72}$ an initial rapid aggregation into percolating clusters was shown, followed by a much more gradual densification of the chains or columns. Evolution of order parameters parallel and perpendicular to the electric field was also simulated by Tao and Jiang. ${ }^{73}$

Experimentally, the diffuse transmittance of light, in which multiple scattering is taken into account, ${ }^{74,75}$ was applied by Ginder et al. ${ }^{76,77}$ to a commercially available ER suspension, and a time scale for the aggregation of the particles on the order of $10^{-3} \mathrm{~s}$ was reported. Electrophoresis was also found by diffusewave spectroscopy. ${ }^{77}$ In a nearly index-matched single-scattering ER suspension, the slow mode for the segregation chain into column on a time scale of $10^{2} \mathrm{~s}$ was reported by Martin et al..$^{78}$ in a real-time, two-dimensional light scattering study. Furthermore, simultaneous measurements of the diffuse transmitted light intensity and the current passing through a dry ER microsuspension were reported by Tanaka et al. ${ }^{79}$ in response to the electric field. Both responses consisted of plural modes. The optical response was expressed as an exponential function with modes 1 (faster) and 2 (slower), which take place in succession:

$$
\begin{aligned}
& \Delta I(t) / I_{0}=\Sigma\left(\Delta I_{\mathrm{i}} / I_{0}\right)\left[1-\exp \left\{\left(t-t_{\mathrm{i}}\right) / \tau_{\mathrm{i}}\right\}\right], \\
& \Delta I_{\mathrm{i}} / I_{0}=0 \text { for } t_{\mathrm{i}}>t,
\end{aligned}
$$

where $\Delta I_{\mathrm{i}} / I_{0}, t_{\mathrm{i}}$, and $\tau_{\mathrm{i}}(\mathrm{i}=1,2)$ are adjustable parameters. Further, the (fastest) mode 0 was qualitatively found only in the current responses, suggesting particle polarization. Time scales including $\tau_{1}$ on the order of $10^{-3} \mathrm{~s}$ were reported. In Figure 5, the development of the microstructures is proposed.

\section{Development of Microstructures under Shear Flow}

Under shear flow, an exponential growth of the induced stress was observed, ${ }^{12,80}$ the expressions of which were similar to eqs 4 and 5 with two (or three) modes. The faster mode

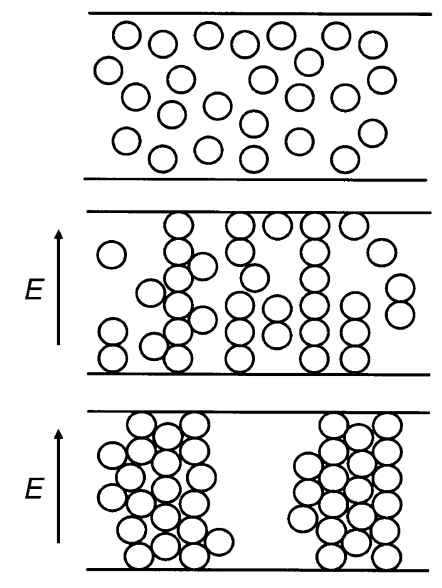

Figure 5. A schematic illustration with Observation 1 shown in Figure 1 for an initial aggregation of polarized particles to form chains (or columns) and later densification of the aggregated chains in the quiescent state.

(mode 1) with $\tau_{1}$ on the order of $10^{-3} \mathrm{~s}$ was proposed to be related to an initial formation of chains (or columns) while the slower mode (mode 2) was related to the yielding of the chains. Transient stress responses to alternating square wave excitations were reported by Ginder and Ceccio. ${ }^{81}$ The characteristic rheological response times for the growth and decay of the stress were observed. An inverse relationship was found between characteristic rheological response times and the shear rate. Biexponential responses have also been reported by Tian et al. ${ }^{82}$ in the tensile stress response.

Furthermore, a scaling behavior of the stress response times $\tau_{\mathrm{i}}(\mathrm{i}=1,2)$ was found by Tanaka et al. ${ }^{12}$ The response time of mode 1 multiplied by the shear rate, $\dot{\gamma} \tau_{1}$, was proportional to $M_{\mathrm{n}}{ }^{0.23}$, while that of mode $2, \dot{\gamma} \tau_{2}$, was almost independent of $M_{\mathrm{n}}$. For mode 1, a many-body effect of particles was closely related to the shear rate dependence of the response time, the relation of which consistently agreed with that obtained in a computer simulation by Takimoto et al. ${ }^{83}$ and confirmed further by experiments. ${ }^{84,85}$ For mode $2, \tau_{2}$ was roughly proportional to $\dot{\gamma}^{-1}$ alone, the relation of which also consistently agreed with the inverse relationship ${ }^{81}$ discussed above. Mode 1 of the stress response is similar to that of the optical response in the quiescent state, suggesting an initial formation of chains (or columns) shown in Figure 5 for $\tau_{1} \ll \dot{\gamma}^{-1}$. On the other hand, shearing effects on the microstructure will be essential for mode 2 .

\section{Microstructures under Shear Flow}

A non-equilibrium phase diagram for model ER suspensions has been reported by Melrose ${ }^{86}$ and Melrose and Heyes ${ }^{87}$ in Brownian dynamics simulations in the space of shear rate $P_{\mathrm{e}}$ and interaction strength $Q$, where $P_{\mathrm{e}}$ is the Peclet number defined as $P_{\mathrm{e}}=3 \pi \eta_{\mathrm{c}} \sigma^{3} \dot{\gamma} / 8 k T$, and $Q=\left(4 \pi k T \varepsilon_{\mathrm{c}} \varepsilon_{0} \sigma^{3}\right)^{-1} \mu^{2}$, with $\sigma$ and $\mu$ respectively defined as the diameter and dipole moment of the particle. ${ }^{86}$ The Peclet number $P_{\mathrm{e}}$ sets the ratio of the shear and Brownian forces. Four distinct phases were observed $\left(Q, P_{\mathrm{e}}\right.$, volume fraction $\phi=0.3$ ). Especially, a layered phase was found, in which the layer structures were developed under shear flow with the layer normal to the vorticity direction. Further- 
more, sheet-like structures were observed by $\operatorname{Martin}^{88}$ in large scale three-dimensional Brownian dynamics simulations. In a two-fluid continuum model by von Pfeil et al. ${ }^{89}$ stripe formation under electric and shear fields was observed.

Experimentally, the effect of an electric field with a shear field was studied by Henley and Filisko ${ }^{90}$ upon microstructures in an ER micro-suspension. Only when the shear and electric fields were applied simultaneously to the ER suspension, lamellar formations were observed, the lamellar structure of which was quenched in a paraffin matrix by cooling. In a fixture of parallel disks, the concentric rings of particles as lamellae appeared along the shear flow. Within the rings, individual spots were also found in Observation 2 shown in Figure 1, suggesting that the spots were related to the ends of the columns and that the rings were formed by the electrically induced columns which were further induced to line up in the rings by the shear flow. Under oscillatory shear and electric fields, stripes of particles were observed earlier by Bossis et al., ${ }^{91}$ the stripes of which were aligned along the flow with a large strain amplitude.

In a Brownian dynamics simulation by Cao et al. $^{92}$ considering the spinning of the dipole moment of the particles under shear flow, the evolution of the microstructure from a random structure to stable lamellar patterns was observed. The corresponding transient stress response was also simulated. On the other hand, layer structures were simulated without the spinning of the dipole moment. ${ }^{86-88}$ Furthermore, the transient rheological response and the evolution of the microstructure have been reported experimentally by Kittipoomwong et al. ${ }^{93}$

\section{YIELDING OF THE MICROSTRUCTURES}

\section{Yield Behavior of Micro-Suspensions}

For an ER suspension based on alumino-silicate particles under sinusoidal strains, three rheological regions were proposed by Gamota and Filisko, ${ }^{94}$ such as pre-yield, yield, and post-yield regions. In the Bingham-body-type model, the importance of the pre-yield region, in which a linear viscoelastic response to the sinusoidal strain was observed, was completely overlooked. The linear viscoelastic response was changed to a nonlinear viscoelastic or viscoelastic plastic behavior as the strain increased. In the post-yield region, the waveform of the stress was found to be significantly distorted. The yield region was also proposed, in which the transient portion of the onset of flow or permanent deformation may be characterized by a yield strain and/or a yield stress.

Strain dependence of the dynamic viscoelastic properties was also studied, ${ }^{33,80,95}$ and linear and non-linear stress responses were observed for suspensions based on cation exchange resin particles or $\mathrm{Fe}(\mathrm{OH})_{3}$-coated silica particles. From the non-linear stress responses with distorted waveform, the non-linear viscoelastic functions, $G_{1}{ }^{\prime}$ and $G_{1}{ }^{\prime \prime}$ derived by Onogi et al., ${ }^{96}$ were calculated using the amplitude of the stress response $\sigma_{1}$ for the sinusoidal wave of the first order. With sufficiently rigid torque sensors, strain dependences of the dynamic moduli for the linear and non-linear responses were obtained, and the yielding process with three steps, two plateau regions and two or three critical strains for yielding, was observed. ${ }^{33,95}$ For the suspension based on cation exchange resin particles, a master curve was obtained ${ }^{95}$ for vertically shifted stresses $b_{\mathrm{E}} \sigma_{1}$ as a double-valued function of $\gamma_{\mathrm{a}}\left(4-\gamma_{\mathrm{a}}^{2}\right) /\left(1+\gamma_{\mathrm{a}}^{2}\right)^{7 / 2}$, where $b_{\mathrm{E}}$ is the shift factor (reduced to the stress at $1 \mathrm{kV} \cdot \mathrm{mm}^{-1}$ ), and $\gamma_{\mathrm{a}}$ is the amplitude of the strain of $3.14 \mathrm{rad} \cdot \mathrm{s}^{-1}$.

Creep behavior was studied by Otsubo and Edamura ${ }^{97,98}$ for suspensions based on composite particles. At both low and high stresses, the suspensions cannot be classified as elastic solids even in the pre-yield region but as liquids. On the other hand, the strain changed almost instantaneously and reached equilibrium at intermediated stresses. Although the retarded strain and viscous flow were negligibly small compared with the instantaneous strain, the strain was not recovered after the removal of the stress, the behavior of which is plastic with an infinite relaxation time. The instantaneous strain without recovery cannot be explained by the single-chain model often assumed. The shear-induced changes of the thick column from an ordered lattice ${ }^{42}$ to another metastable configuration were proposed for the plastic response.

\section{Dip Behavior of Micro-Suspensions}

Although a typical flow behavior has been experimentally reported for an ER suspension based on cation exchange resin particles under steady shear flow, ${ }^{99}$ rather complicated flow behavior has also been reported by Tanaka et al. for similar suspensions. ${ }^{99-101}$ For $M_{\mathrm{n}}>0.3$, the shear thinning was found for a suspension with a particle concentration of $30 \mathrm{wt} \%$ $(\phi=0.33),{ }^{101}$ the particles of which were suspended in a silicone oil with higher viscosity $\eta_{\mathrm{c}}$. Further, a remarkable dip behavior was found for suspensions of 5 and $10 \mathrm{wt} \%$ : The stress decreased with an increase in the shear rate, keeping $\sigma(E)>\sigma(0)$, and it gradually increased again and approached the stress under no electric fields. ${ }^{99-101}$ Behavior similar to the dip behavior (or shear thinning) was often reported for other suspensions. ${ }^{102,103}$ The dip behavior was sensitively probed by the apparent conductivity, which was measured simultaneously with stress. ${ }^{99-101}$ The apparent conductivity also showed a steep decrease above $M_{\mathrm{n}} \sim 10^{-2}$ for the suspensions of 5 and $10 \mathrm{wt} \%$. Interestingly, the apparent conductivity for the suspension of $5 \mathrm{wt} \%$ showed not only a local minimum around $M_{\mathrm{n}} \sim 10^{-1}$ but also a local maximum after the minimum, the local maximum of which was not observed ${ }^{100}$ in the stress scaled by $2 \varepsilon_{\mathrm{c}} \varepsilon_{0}(\beta E)^{2}$. The dynamic yield stress, $\sigma_{\mathrm{y}}(E)$, at a given electric field was not necessarily constant above a critical value of $M_{\mathrm{n}}$. The dip behavior is closely related to a balance between fracture and recombination of polarized particles or clusters under shear flow, the behavior of which is not easily confirmed directly using optical microscopy.

\section{Yield Behavior of Nano-Suspensions}

The flow behavior of a suspension composed of dry $\mathrm{TiO}_{2}$ nano-particles in silicone oil $\left(\varepsilon_{\mathrm{r}}=2.7\right)$ with a primary particle diameter $(2 a)$ of $15 \mathrm{~nm}$ and $\phi=0.088$ has been studied at room temperature. ${ }^{37}$ The stress induced by a DC (as well as sinusoidal) electric field was mostly proportional to the square 
of the electric field of $E$. The $E^{2}$ dependence of the dynamic storage modulus $G^{\prime}$ was also found ${ }^{26}$ above $2 \mathrm{kV} \cdot \mathrm{mm}^{-1}$. Below $2 \mathrm{kV} \cdot \mathrm{mm}^{-1}, G^{\prime}$ was larger than the line showing the $E^{2}$ dependence. The upward deviation from the $E^{2}$ dependence would be caused by the particle-particle interaction without external electric fields. In optical observations with Observation 1 shown in Figure 1, a fine chain-like microstructure was found in the quiescent state under the DC electric field. The electrically induced chain-like microstructure will be responsible for the ER effect of the nano-suspension, which is apparently similar to the micro-suspension. However, the ER effect was unexpectedly induced by a strength of the electric field on the order of $10^{6} \mathrm{~V} \cdot \mathrm{m}^{-1}$ at room temperature, which was much lower than expected based on the primary particle using $P\left[=k T / a^{3} 4 \pi \varepsilon_{\mathrm{c}} \varepsilon_{0}(\beta E)^{2}\right]$. Furthermore, the nano-suspension showed an apparent yield stress around $10 \mathrm{~Pa}$ as well as non-zero values of $G^{\prime}$ on the order of $10^{2} \mathrm{~Pa}$ even under no electric fields. ${ }^{26,37}$ The dynamic loss modulus $G^{\prime \prime}$ as well as $G^{\prime}$ was almost independent of the frequency, and $G^{\prime}$ was larger than $G^{\prime \prime}$ in the absence (or in the presence) of electric field. ${ }^{26}$

For the micro-suspension under no electric fields, $G^{\prime}$ is essentially zero because the flow is assumed to be Newtonian. ${ }^{97-99,104-106}$ However, a plateau region with a stress smaller than $10^{-3} \mathrm{~Pa}$ was observed by Marshall et al. ${ }^{43}$ at shear rates below $10^{-2} \mathrm{~s}^{-1}$ under no electric fields for a suspension of weakly flocculated non-Brownian particles with $\phi=0.13$. A slightly pseudoplastic flow was also observed by Otsubo et $a .^{107}$ at lower shear rates under no electric fields for a suspension with sub-micrometer particles of monodispersed silica with $\phi=0.4$, while no elastic responses in the dynamic measurement were shown without electric fields. ${ }^{98,105,107} \mathrm{~A}$ small but non-zero value of $G^{\prime}$ around $1 \mathrm{~Pa}$ was preliminarily observed $^{95}$ under no electric fields at an angular frequency of $3.14 \mathrm{rad} \cdot \mathrm{s}^{-1}$ for a suspension based on cation exchange resin particles of $\phi=0.33$, suggesting a weak flocculation of nonBrownian particles. In a Brownian dynamics simulation, nonzero value of high-frequency shear modulus under no electric fields was shown by Adriani and Gast. ${ }^{48}$

In the presence of electric field, the $E^{2}$ dependence of the shear modulus for a perfectly elastic material was reported by Jordan et $a l .{ }^{108}$ for non-Brownian suspensions. In a dimensional analysis, a time-field strength superposition principle was proposed for non-Brownian suspensions by Parthasarathy et al. ${ }^{105}$ For Brownian suspensions, ${ }^{48}$ a gradual increase in high-frequency shear modulus with $E$ was simulated. Similar results of shear moduli were also predicted by Whittle. ${ }^{109}$

In the frequency dependence of $G^{\prime}$ (or $G^{\prime \prime}$ ), a plateau was experimentally observed under an electric field for a microsuspension based on composite particles. ${ }^{98}$ Frequency dependent dynamic moduli were reported by Conrad et al. ${ }^{104}$ for a micro-suspension based on corn starch particles. Strongly frequency-dependent behavior of $G^{\prime}$ was also observed..$^{98,105}$ Furthermore, the Maxwell-type relaxation was observed under an electric field by Otsubo et al. ${ }^{107}$ at very small strains for the suspensions based on sub-micrometer particles with $\phi=0.2$ and 0.4 .

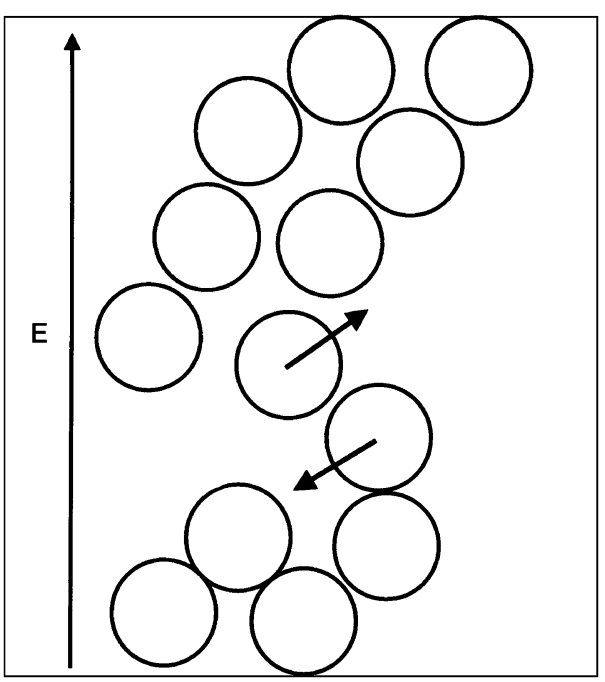

Figure 6. A schematic illustration for thermal fluctuations of secondary particles within a chain-like microstructure including some imperfections. Rearrangement of particles can be induced by the Brownian fluctuations.

The energy dissipation in the dynamic measurements for the ER micro-suspensions was explained by the hydrodynamic interactions with drifting chains, and chains attached at only one end to an electrode. ${ }^{105-108} \mathrm{~A}$ significant and sharp relaxation was simulated for a thick cluster composed of a single-sphere-width strand with two additional strands placed along each side of the original strand. ${ }^{105}$ In a model considering particle-strings between parallel electrodes, ${ }^{106}$ a plateau in $G^{\prime}$ was expected. A plateau-like region in $G^{\prime \prime}$ was also developed at intermediate frequencies as the string length increased. In the nano-suspension, both $G^{\prime}$ and $G^{\prime \prime}$ at a given strength of the electric field were mostly independent of frequency. The energy dissipation would also be induced by the Brownian motion of secondary particles within each chain-like microstructure of nano-particles including some imperfections, as shown in Figure 6, because the width of the microstructure was on the order of micrometers and much wider than the diameter of the primary particle. ${ }^{26}$ In the imperfect microstructure, rearrangement of particles can be induced by the Brownian fluctuations, so that the energy dissipation is independent of the frequency of oscillatory strain.

Under no electric fields, it is considered that a microstructure, such as a three-dimensional network, ${ }^{27}$ was developed by the particle-particle interaction. Such a network microstructure has been observed optically ${ }^{110}$ for a nanosuspension based on nano-particles of $\mathrm{TiO}_{2}$ with $\phi=0.024$, the nano-suspension of which also showed an apparent yield stress around $1 \mathrm{~Pa}$ under no electric fields. On the other hand, no plateau region was measured for the nano-suspension with $\phi=0.012$, and secondary particles on the order of several micrometers were observed to be separately distributed. The microstructure (or secondary particles) developed under no electric fields can essentially be related to not only the stability against sedimentation and the flow behavior under no electric fields, but also to the low strength of the electric field for the ER effect. It is proposed that randomly distributed secondary 
particles or a macroscopically developed microstructure of secondary particles in the nano-suspension should also be taken into account for the initial state of the ER effect, other than randomly distributed primary particles generally assumed so far.

\section{CONCLUDING REMARKS}

Electrically induced microstructures, yielding, and related physical properties have been extensively studied in terms of theory and experiment. The microstructures can be sensitively probed by rheology and related physical properties, the development and yielding of which are not easily confirmed directly using optical microscopy. The fundamental properties observed experimentally can be qualitatively explained by the models proposed. However, there are still demands for the ER fluids to be improved. Although a much larger yield stress has been a major demand, stability in the ER effect would also be a demand for practical applications. Further progress is expected in the field of electrorheology.

Acknowledgment. One of the authors (K. T.) is grateful to Prof. Qui Tran-Cong-Miyata of Kyoto Institute of Technology for providing the opportunity to review electrorheology.

Received: August 6, 2009 Accepted: September 4, 2009 Published: October 21, 2009

\section{REFERENCES}

1. W. M. Winslow, U. S. Patent 2,417,850 (1947).

2. W. M. Winslow, J. Appl. Phys., 20, 1137 (1949).

3. D. L. Klass and T. W. Martinek, J. Appl. Phys., 38, 67 (1967).

4. D. L. Klass and T. W. Martinek, J. Appl. Phys., 38, 75 (1967).

5. N. Sugimoto, Bull. JSME, 20, 1476 (1977).

6. J. E. Stangroom, Phys. Technol., 14, 290 (1983).

7. A. F. Sprecher, J. D. Carlson, and H. Conrad, Mater. Sci. Eng., 95, 187 (1987).

8. H. Block, J. P. Kelly, A. Qin, and T. Watson, Langmuir, 6, 6 (1990).

9. F. E. Filisko and L. H. Radzilowski, J. Rheol., 34, 539 (1990).

10. D. J. Klingenberg and C. F. Zukoski, Langmuir, 6, 15 (1990).

11. A. Hosseini-Sianaki, W. A. Bullough, R. Firoozian, J. Makin, and R. C. Tozer, Int. J. Mod. Phys. B, 6, 2667 (1992).

12. K. Tanaka, A. Sahashi, R. Akiyama, and K. Koyama, Phys. Rev. E: Stat., Nonlinear, Soft Matter Phys., 52, R3325 (1995).

13. J. Furusho and M. Sakaguchi, Int. J. Mod. Phys. B, 13, 2151 (1999).

14. M. Nakano, S. Minagawa, and K. Hagino, Int. J. Mod. Phys. B, 13, 2168 (1999).

15. K. Tanaka, Kobunshi, 47, 542 (1998).

16. K. Tanaka, in "Polymer Yearbook," No. 15, R. A. Pethrick, G. E. Zaikov, T. Tsuruta, and N. Koide, Ed., Harwood Academic Pub., 1998, pp 273-277.

17. H. Block and J. P. Kelly, J. Phys. D: Appl. Phys., 21, 1661 (1988).

18. T. C. Jordan and M. T. Shaw, IEEE Trans. Electric. Insulation, 24, 849 (1989).

19. "Proc. of 2nd Int. Conf. on ER Fluids," J. D. Carlson, A. F. Sprecher, and H. Conrad, Ed., Technomic Publishing, Lancaster, 1990, pp 1-454.

20. "Proc. of 3rd Int. Conf. on ER Fluids," R. Tao, Ed., World Scientific, Singapore, 1992, pp 1-502.

21. "Progress in Electrorheology," K. O. Havelka and F. E. Filisko, Ed., Plenum Press, New York, 1995, pp 1-372.

22. "Proc. of 6th Int. Conf. on ER Fluids, MR Suspensions and Their
Applications," M. Nakano and K. Koyama, Ed., World Scientific, Singapore, 1998, pp 1-893.

23. T. C. Halsey, Science, 258, 761 (1992).

24. M. Parthasarathy and D. J. Klingenberg, Mater. Sci. Eng., R17, 57 (1996).

25. A. M. Kraynik, in "Proc. of 2nd Int. Conf. on ER Fluids," J. D. Carlson, A. F. Sprecher, and H. Conrad, Ed., Technomic Publishing, 1990, pp 445-454.

26. K. Tanaka, H. Nakahori, K. Katayama, and R. Akiyama, Colloid Polym. Sci., 285, 1201 (2007).

27. T. Matsumoto, C. Hitomi, and S. Onogi, Trans. Soc. Rheol., 19, 541 (1975).

28. H. A. Barnes and K. Walters, Rheol. Acta, 24, 323 (1985).

29. T. Shiga, M. Fujimoto, Y. Hirose, A. Okada, and T. Kurauchi, Nihon Reoroji Gakkaishi, 20, 85 (1992).

30. K. Koyama, K. Minagawa, T. Yoshida, N. Kuramoto, and K. Tanaka, Mod. Phys. Lett. B, 8, 1563 (1994).

31. Y. Ishino, T. Maruyama, T. Ohsaki, S. Endo, T. Saito, and N. Goshima, in "Progress in Electrorheology," K. O. Havelka and F. E. Filisko, Ed., Plenum Press, 1995, pp 137-146.

32. A. Inoue, in "Proc. of 2nd Int. Conf. on ER Fluids," J. D. Carlson, A. F. Sprecher, and H. Conrad, Ed., Technomic Publishing, 1990, pp 176-183.

33. K. Tanaka, K. Koyama, and T. Watanabe, in "Progress in Electrorheology,” K. O. Havelka and F. E. Filisko, Plenum Press, 1995, pp 273-279.

34. N. Kuramoto, M. Yamazaki, K. Nagai, K. Koyama, K. Tanaka, K. Yatsuzuka, and Y. Higashiyama, Thin Solid Films, 239, 169 (1994).

35. N. Kuramoto, M. Yamazaki, K. Nagai, K. Koyama, K. Tanaka, K. Yatsuzuka, and Y. Higashiyama, Rheol. Acta, 34, 298 (1995).

36. Y. Asako, S. Ono, R. Aizawa, and T. Kawakami, in "Progress in Electrorheology,” K. O. Havelka and F. E. Filisko, Plenum Press, 1995, pp 147-156.

37. K. Tanaka, T. Wakayasu, A. Kubono, and R. Akiyama, Sens. Actuators, A, 112, 376 (2004).

38. K. Lozano, C. Hernandez, T. W. Petty, M. B. Sigman, and B. Korgel, J. Colloid Interface Sci., 297, 618 (2006).

39. M. Lizcano, M. R. Nava-Lara, A. Alvarez, and K. Lozano, Carbon, 45, 2374 (2007).

40. J. Yin and X. Zhao, J. Phys. D: Appl. Phys., 34, 2063 (2001).

41. W. Wen, X. Huang, S. Yang, K. Lu, and P. Sheng, Nat. Mater., 2, 727 (2003).

42. R. Tao and J. M. Sun, Phys. Rev. Lett., 67, 398 (1991).

43. L. Marshall, C. F. Zukoski, and J. W. Goodwin, J. Chem. Soc. Faraday Trans. 1, 85, 2785 (1989).

44. W. B. Russel, J. Rheol., 24, 287 (1980).

45. H. See, H. Tamura, and M. Doi, J. Phys. D: Appl. Phys., 26, 746 (1993).

46. P. A. Arp, R. T. Foister, and S. G. Mason, Adv. Colloid Interface Sci., 12, 295 (1980).

47. J. Takimoto, Nihon Reoroji Gakkaishi, 20, 95 (1992).

48. P. M. Adriani and A. P. Gast, Phys. Fluids, 31, 2757 (1988).

49. T. C. Halsey and W. Toor, Phys. Rev. Lett., 65, 2820 (1990).

50. T. C. Halsey, J. E. Martin, and D. Adolf, Phys. Rev. Lett., 68, 1519 (1992).

51. D. J. Klingenberg, F. Van Swol, and C. F. Zukoski, J. Chem. Phys., 91, 7888 (1989).

52. H. See and M. Doi, J. Phys. Soc. Jpn., 60, 2778 (1991).

53. D. J. Klingenberg, F. Van Swol, and C. F. Zukoski, J. Chem. Phys. 94, 6160 (1991).

54. J. Takimoto, in "Proc. of 3rd Int. Conf. on ER Fluids," R. Tao, Ed., World Scientific, 1992, pp 53-58.

55. R. T. Bonnecaze and J. F. Brady, J. Chem. Phys., 96, 2183 (1992).

56. R. T. Bonnecaze and J. F. Brady, J. Rheol., 36, 73 (1992).

57. M. E. Gurtin, "An Introduction to Continuum Mechanics," Academic Press, New York, 1981, pp 1-265.

58. R. A. Anderson, in "Proc. of 3rd Int. Conf. on ER Fluids," R. Tao, Ed., World Scientific, 1992, pp 81-90. 
59. L. C. Davis, J. Appl. Phys., 72, 1334 (1992).

60. L. C. Davis, J. Appl. Phys., 73, 680 (1993).

61. P. J. Rankin and D. J. Klingenberg, J. Rheol., 42, 639 (1998).

62. J.-N. Foulc, P. Atten, and N. Félici, J. Electrost., 33, 103 (1994).

63. L. Onsager, J. Chem. Phys., 2, 599 (1934).

64. X. Tang, C. Wu, and H. Conrad, J. Rheol., 39, 1059 (1995).

65. K. Negita and Y. Ohsawa, Phys. Rev. E: Stat., Nonlinear, Soft Matter Phys., 52, 1934 (1995).

66. Y. Misono and K. Negita, Phys. Rev. E: Stat., Nonlinear, Soft Matter Phys., 70, 061412 (2004).

67. T. C. Halsey, in "Proc. of 3rd Int. Conf. on ER Fluids," R. Tao Ed., World Scientific, 1992, pp 37-52.

68. R. Tao and J. M. Sun, Phys. Rev. A: At., Mol., Opt. Phys., 44, R6181 (1991).

69. T. Chen, R. N. Zitter, and R. Tao, Phys. Rev. Lett., 68, 2555 (1992).

70. G. L. Gulley and R. Tao, Phys. Rev. E: Stat., Nonlinear, Soft Matter Phys., 56, 4328 (1997).

71. A. Kumar, B. Khusid, Z. Qiu, and A. Acrivos, Phys. Rev. Lett., 95, 258301-1 (2005)

72. W. R. Toor, J. Colloid Interface Sci., 156, 335 (1993).

73. R. Tao and Q. Jiang, Phys. Rev. Lett., 73, 205 (1994).

74. I. Freund, Phys. Rev. A: At., Mol., Opt. Phys., 45, 8854 (1992).

75. D. J. Durian, D. A. Weitz, and D. J. Pine, Phys. Rev. A: At., Mol., Opt. Phys., 44, R7902 (1991).

76. J. M. Ginder and L. D. Elie, in "Proc. of 3rd Int. Conf. on ER Fluids,” R. Tao, Ed., World Scientific, 1992, pp 23-36.

77. J. M. Ginder, Phys. Rev. E: Stat., Nonlinear, Soft Matter Phys., 47, 3418 (1993).

78. J. E. Martin, J. Odinek, and T. C. Halsey, Phys. Rev. Lett., 69, 1524 (1992).

79. K. Tanaka, K. Nakamura, and R. Akiyama, Phys. Rev. E: Stat., Nonlinear, Soft Matter Phys., 62, 5378 (2000).

80. K. Tanaka, T. Yoshida, and K. Koyama, in "Proc. of 3rd Int. Conf. on ER Fluids,” R. Tao, Ed., World Scientific, 1992, pp 289-299.

81. J. M. Ginder and S. L. Ceccio, J. Rheol., 39, 211 (1995).

82. Y. Tian, H. Zeng, T. H. Anderson, B. Zhao, P. McGuiggan, and J. Israelachvili, Phys. Rev. E: Stat., Nonlinear, Soft Matter Phys., 75, 011409 (2007)

83. J. Takimoto, K. Minagawa, and K. Koyama, Int. J. Mod. Phys. B, 10, 3037 (1996).

84. K. Tanaka, K. Nakamura, K. Takada, F. Iwaki, A. Kubono, R. Akiyama, and N. Kuramoto, Int. J. Mod. Phys. B, 13, 1814 (1999).

85. K. Tanaka, S. Hashimoto, T. Takenouchi, I. Sugimoto, A. Kubono, and R. Akiyama, Int. J. Mod. Phys. B, 15, 930 (2001).

86. J. R. Melrose, Phys. Rev. A: At., Mol., Opt. Phys., 44, R4789 (1991).

87. J. R. Melrose and D. M. Heyes, J. Chem. Phys., 98, 5873 (1993).

88. J. E. Martin, Phys. Rev. E: Stat., Nonlinear, Soft Matter Phys., 63, 011406 (2000).

89. K. von Pfeil, M. D. Graham, D. J. Klingenberg, and J. F. Morris, Phys. Rev. Lett., 88, 188301 (2002).

90. S. Henley and F. E. Filisko, J. Rheol., 43, 1323 (1999).

91. G. Bossis, Y. Grasselli, E. Lemaire, L. Petit, and J. Persello, in "Proc. of 3rd Int. Conf. on ER Fluids," R. Tao, Ed., World Scientific, 1992, pp 75-80.

92. J. G. Cao, J. P. Huang, and L. W. Zhou, J. Phys. Chem. B, 110, 11635 (2006)

93. D. Kittipoomwong, D. J. Klingenberg, Y. M. Shkel, J. F. Morris, and J. C. Ulicny, J. Rheol., 52, 225 (2008).

94. D. R. Gamota and F. E. Filisko, J. Rheol., 35, 399 (1991).

95. K. Tanaka, S. Yamamoto, R. Akiyama, and K. Koyama, Nihon Reoroji Gakkaishi, 25, 31 (1997).

96. S. Onogi, T. Masuda, and T. Matsumoto, Trans. Soc. Rheol., 14, 275 (1970)

97. Y. Otsubo and K. Edamura, J. Rheol., 38, 1721 (1994).

98. Y. Otsubo and K. Edamura, J. Colloid Interface Sci., 172, 530 (1995).

99. K. Tanaka, T. Takenouchi, and R. Akiyama, in "Proc. of 26th Ann. Conf. of IEEE," S. Okuma, IEEE, 2000, pp 1821-1826.

100. K. Tanaka, K. Ichizawa, R. Akiyama, and A. Kubono, Nihon Reoroji Gakkaishi, 29, 105 (2001).

101. K. Tanaka, K. Ichizawa, Y. Onishi, A. Kubono, and R. Akiyama, Int. J. Mod. Phys. B, 16, 2683 (2002).

102. H. See, A. Kawai, and F. Ikazaki, Colloid Polym. Sci., 280, 24 (2002).

103. S. G. Kim, J. Y. Lim, J. H. Sung, H. J. Choi, and Y. Seo, Polymer, 48, $6622(2007)$

104. H. Conrad, Y. Li, and Y. Chen, J. Rheol., 39, 1041 (1995).

105. M. Parthasarathy, K. H. Ahn, B. M. Belongia, and D. J. Klingenberg, Int. J. Mod. Phys. B, 8, 2789 (1994).

106. T. C. B. McLeish, T. Jordan, and M. T. Shaw, J. Rheol., 35, 427 (1991).

107. Y. Otsubo, M. Sekine, and S. Katayama, J. Rheol., 36, 479 (1992).

108. T. C. Jordan, M. T. Shaw, and T. C. B. McLeish, J. Rheol., 36, 441 (1992).

109. M. Whittle, J. Non-Newtonian Fluid Mech., 37, 233 (1990).

110. K. Tanaka, N. Sezaki, H. Nakahori, and R. Akiyama, Nihon Reoroji Gakkaishi, 37, 17 (2009).

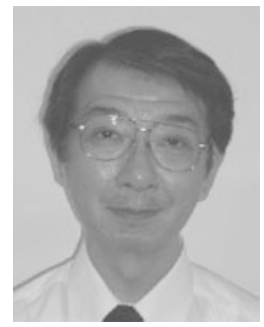

Katsufumi Tanaka was born in Tokyo, Japan, and received his B.Eng., M.Eng. and D.Eng. degrees in Organic and Polymeric Materials from Tokyo Institute of Technology, Tokyo, Japan, in 1983, 1985 and 1988, respectively. He is currently an Associate Professor in Kyoto Institute of Technology, Kyoto, Japan, where he moved from Yamagata University in 1992. He was awarded Takeshi Sakurada Memorial Award of The Society of Fiber Science and Technology, Japan in 1992, and Award for outstanding paper, Nihon Reoroji Gakkaishi (J. Soc. Rheol., Jpn.) in 2003. He was a member of reviewers of Polymer Yearbook. He is currently a member of associate editors of Nihon Reoroji Gakkaishi, and a member of representatives of The Society of Rheology, Japan. His research interests cover physical properties of polymers; microstructure and physical properties of complex fluids in response to external modulation of stimuli, including electro-rheology of liquid crystalline polymers and suspensions based on micro- or nano-particles.

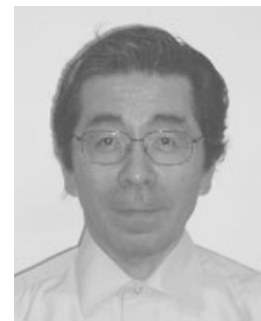

Ryuichi Akiyama was born in Kanagawa, Japan. He received his B.Eng., M.Eng. and D.Eng. degrees in Textile Engineering from Tokyo Institute of Technology, Tokyo, Japan, in 1972, 1974 and 1983, respectively. He is currently a Professor in Kyoto Institute of Technology, Kyoto, Japan, where he joined as a faculty in 1982. He was awarded Takeshi Sakurada Award of The Society of Fiber Science and Technology, Japan in 1983. He is interested in liquid crystals, sensory test of fabrics, and electro-rheology. 